\title{
Spatiotemporal Gating of Sensory Inputs in Thalamus during Quiescent and Activated States
}

\author{
Juan R. Aguilar and Manuel A. Castro-Alamancos \\ Department of Neurobiology and Anatomy, Drexel University College of Medicine, Philadelphia, Pennsylvania 19129
}

\begin{abstract}
The main role of the thalamus is to relay sensory inputs to the neocortex according to the regulations dictated by behavioral state. Hence, changes in behavioral state are likely to transform the temporal and spatial properties of thalamocortical receptive fields. We compared the receptive fields of single cells in the ventroposterior medial thalamus (VPM) of urethane-anesthetized rats during quiescent states and during aroused (activated) states. During quiescent states, VPM cells respond to stimulation of a principal whisker (PW) and may respond modestly to one or a few adjacent whiskers (AWs). During either generalized forebrain activation or selective thalamic activation caused by carbachol infusion in the VPM, the responses to AWs enhance so that VPM receptive fields become much larger. Such enlargement is not observed at the level of the principal trigeminal nucleus, indicating that it originates within the thalamus. Interestingly, despite the increase in AW responses during activation, simultaneous deflection of the PW and AWs produced VPM responses that resembled the PW response, as if the AWs were not stimulated. This nonlinear summation of sensory responses was present during both quiescent and activated states. In conclusion, the thalamus suppresses the excitatory surround (AWs) of the receptive field during quiescent states and enlarges this surround during arousal. But, thalamocortical cells represent only the center (PW) of the receptive field when the center (PW) and surround (AWs) are stimulated simultaneously.
\end{abstract}

Key words: thalamus; neocortex; vibrissas; sensory processing; vigilance; attention; brainstem reticular formation; acetylcholine

\section{Introduction}

In the rodent vibrissa system (Woolsey and Van der Loos, 1970; Bernardo and Woolsey, 1987), a whisker deflection drives mechanoreceptors on the sensory axon of a trigeminal ganglion cell, triggering activity that ascends toward the neocortex via the trigeminal complex, where groups of cells form cell clusters called "barrelettes" (Henderson and Jacquin, 1995). The axons of these trigeminal cells produce the lemniscal pathway that innervates clusters of cells in the ventroposterior medial thalamus (VPM), called "barreloids" (Land et al., 1995). Finally, the axons of these VPM cells produce a thalamocortical pathway that innervates clusters of cells in layer IV, called "barrels" (Woolsey and Van der Loos, 1970). Rats use this sensory system to navigate and to locate and identify objects in their environment (Guic-Robles et al., 1989; Carvell and Simons, 1990; Brecht et al., 1997).

At the center of processing and response transformation of sensory information reaching the neocortex is the lemniscal or trigeminothalamic pathway. Indeed, the output produced by this synaptic connection is the input of the barrel cortex. Recent work has shown that important transformations occur at the lemniscal

\footnotetext{
Received May 31, 2005; revised 0ct. 16, 2005; accepted 0ct. 18, 2005.

This work was supported by the National Institutes of Health and the EJLB Foundation. J.R.A. was supported in part by a postdoctoral fellowship from the Ministry of Science and Education of Spain. We thank Yoshie TawaraHirata for excellent technical support and Akio Hirata and Jeremy Cohen for comments on this manuscript.

More information can be found at http://neurobio.drexelmed.edu/CastroWeb/castro.html.

Correspondence should be addressed to Dr. Manuel A. Castro-Alamancos, Department of Neurobiology and Anatomy, Drexel University College of Medicine, 2900 Queen Lane, Philadelphia, PA 19129. E-mail: mcastro@drexelmed.edu.

DOI:10.1523/JNEUROSCI.3229-05.2005

Copyright $\odot 2005$ Society for Neuroscience $\quad$ 0270-6474/05/2510990-13\$15.00/0
}

pathway as a function of timing of the sensory input and as a consequence of changes in behavioral state (for review, see Moore, 2004; Castro-Alamancos, 2004b). There is also evidence suggesting that important spatial receptive field transformations may take place at this connection. For instance, previous studies that recorded from VPM neurons in anesthetized rats indicated that they respond solely to a single whisker (Waite, 1973; Shosaku, 1985; Rhoades et al., 1987; Chiaia et al., 1991). However, more recent work has revealed that under light anesthesia, VPM cells respond to deflections of multiple whiskers, including a short-latency principal whisker (PW) plus several adjacent whiskers (AWs) that respond with longer latencies (Simons and Carvell, 1989; Armstrong-James and Callahan, 1991; Diamond et al., 1992; Nicolelis and Chapin, 1994; Friedberg et al., 1999; Brecht and Sakmann, 2002; Minnery et al., 2003). One possibility is that the size of the receptive field can be modified as a function of the level of arousal, so that during nonactivated (quiescent) states, VPM neurons respond mostly to one whisker (PW), whereas during activated states, the same VPM neuron responds to a PW and to a number of AWs.

Arousal in behaving animals is accompanied by an electrographic sign called activation, which consists of a low-amplitude fast field potential activity recorded from the forebrain (Moruzzi and Magoun, 1949; Vanderwolf, 1988). This activity contrasts with the large-amplitude slow oscillatory activity typical of quiescent or nonactivated states. The thalamus is particularly susceptible to changes in activation (Steriade et al., 1997). In the rat, thalamic activation is accompanied by a robust increase in the firing rate of VPM neurons (Castro-Alamancos, 2004b). Moreover, during thalamic activation, the relay of high-frequency sen- 
sory inputs to the neocortex is allowed, which is normally impeded during quiescent states (Castro-Alamancos, 2002b). Considering the significant effects of activation on the temporal response properties of VPM cells, the present study investigated the consequences of activation on their spatial response properties.

\section{Materials and Methods}

Surgery. Fifty-six adult Spague Dawley rats (300-350 g) were used in this study and cared for in accordance with National Institutes of Health guidelines for laboratory animal welfare. All experiments were approved by the Drexel University Institutional Animal Care and Use Committee. Rats were anesthetized with urethane $(1.5 \mathrm{~g} / \mathrm{kg}$, i.p.) and placed in a stereotaxic frame. All skin incisions and frame contacts with the skin were injected with lidocaine (2\%). A unilateral craniotomy extended over a large area of the parietal cortex. Small incisions were made in the dura as necessary, and the cortical surface was covered with artificial CSF (ACSF) containing the following (in $\mathrm{mm}$ ): $126 \mathrm{NaCl}, 3 \mathrm{KCl}, 1.25$ $\mathrm{NaH}_{2} \mathrm{PO}_{4}, 26 \mathrm{NaHCO}_{3}, 1.3 \mathrm{MgSO}_{4} 7 \mathrm{H}_{2} \mathrm{O}, 10$ dextrose, and $1 \mathrm{CaCl}_{2}$ $2 \mathrm{H}_{2} \mathrm{O}$ or 1 saline. Body temperature was automatically maintained constant with a heating pad at $37^{\circ} \mathrm{C}$. The level of anesthesia was monitored with field recordings and limb-withdrawal reflexes and kept constant at about stage III/3 using supplemental doses of urethane (Friedberg et al., 1999), unless indicated otherwise.

Electrophysiology. Single-unit recordings in the VPM were performed as described previously (Castro-Alamancos, 2002b), using electrodes pulled from glass pipettes $(10-30 \mathrm{M} \Omega)$ that were filled with ACSF or saline. These electrodes generally record only a well discernible single unit of very large amplitude ( $>10$ times the noise). Every cell included in this study corresponds to a recording in which there was only one discernable large-amplitude spike in the recording electrode. Several attempts were made to label these cells using juxtacellular procedures, with some success (see below, Histology). Also, a tungsten electrode was placed in the depth $(0.5-1 \mathrm{~mm})$ of the frontal agranular (motor) neocortex to record spontaneous field potential activity (filter settings, $0.7-170$ $\mathrm{Hz}$ ) and thus to monitor the depth of anesthesia. This electrode was placed in the hemisphere contralateral to the stimulated whiskers. Recordings from the principal nucleus of the trigeminal complex (Pr5) were obtained using a tungsten electrode $(2-6 \mathrm{M} \Omega$ ) placed ipsilateral to the stimulated whiskers. In some cases, recordings were performed simultaneously from VPM and Pr5 cells. In other cases, a bipolar concentric stimulating electrode (total diameter, $200 \mu \mathrm{m}$ ) was placed in the VPM to antidromically identify the VPM-projecting Pr5 cells. A Pr5 cell was considered to project to the VPM if the VPM stimulation evoked a spike that had a constant latency $(<0.1 \mathrm{~ms}$ jitter $)$ and followed high-frequency stimulation $(50-100 \mathrm{~Hz}, 5-10$ pulses). Additional evidence was obtained by performing a collision test. In this case, the antidromic stimulus was triggered by a spontaneous Pr5 spike at a specific interval. A successful collision between the Pr5 spike and the antidromic spike was final evidence that the cell projected to the VPM. The collision should occur for intervals below the sum of the antidromic spike latency plus the axonal refractory period (estimated with paired pulses). The positioning of the VPM antidromic stimulating electrode was guided by the coordinates shown below and by recording field potential responses evoked in the barrel cortex. Moreover, the placement in the VPM was later confirmed histologically.

Recordings and stimulation in the thalamus were obtained from the VPM contralateral to the stimulated whiskers at approximately the following coordinates (Paxinos and Watson, 1982) from bregma: posterior, 3.5; lateral, 3; depth, 5-6. Recordings from the Pr5 ipsilateral to the stimulated whiskers were obtained by inserting the recording electrode at an $\sim 15^{\circ}$ angle (with respect to the vertical plane) in the anteroposterior direction. The coordinates for electrode insertion were approximately as follows: posterior, 7; lateral, 3. The electrode was lowered $\sim 9 \mathrm{~mm}$. Recordings were acquired using a multichannel acquisition processor (MAP) system (Plexon, Dallas, TX).

Reticular formation stimulation. One of the methods used to produce activation in the anesthetized rat was to stimulate the brainstem reticular formation (RF), as described previously (Castro-Alamancos, 2002b; Castro-Alamancos and Oldford, 2002). RF stimulation was delivered using a bipolar stimulating electrode $(200 \mu \mathrm{m}$ diameter concentric; Frederick Haer Company, Bowdoinham, ME) placed contralateral to the stimulated whiskers. The coordinates for RF stimulation were as follows: posterior, 9; lateral, 0.7; depth, 5-6. These coordinates were slightly adjusted so that high-frequency stimulation $(100 \mathrm{~Hz}$ for $1 \mathrm{~s})$ produced robust activation consisting of a depolarizing effect on the recorded VPM cell and a transformation of the cortical field potential activity from a large-amplitude slow activity to a small-amplitude fast activity. The stimulation intensity used was $<200 \mu \mathrm{A}$, and it did not evoke whisker motion.

Microdialysis. As described previously (Castro-Alamancos, 2002b), to apply drugs into the VPM, a microdialysis cannula (250 $\mu$ m diameter, 2 $\mathrm{mm}$ long membrane) was placed around the following coordinates: posterior, 3; lateral, 2-3; depth, $4-6$. The cannula entered into the brain at an angle $\left(\sim 30^{\circ}\right)$ from the midline, whereas the VPM recording electrode entered lateral to the cannula and parallel to the midline (see Fig. $1 \mathrm{~A}$ ). ACSF was infused continuously through the probe at $2-4 \mu \mathrm{l} / \mathrm{min}$. Carbachol was dissolved in the ACSF at $500 \mu \mathrm{M}$.

Whisker stimulation. Sensory stimulation consisted of independently deflecting up to six individual whiskers using six different whisker stimulators. After isolating a unit, the whiskers were stimulated using a handheld probe. The whisker that produced the shortest latency and strongest response was considered the PW. This whisker and up to five additional whiskers surrounding it, called AWs, were selected for stimulation. Each of them was placed in an independent whisker stimulator. Thus, in many cases, six whisker stimulators were used simultaneously, which allowed to independently stimulate the six whiskers. Each of the selected whiskers was inserted into a glass micropipette ( $1 \mathrm{~mm}$ diameter) that was glued to the membrane of a miniature speaker. Application of a $1 \mathrm{~ms}^{2}$ current pulse to the speaker deflected the micropipette and the whiskers inside $(\sim 400 \mu \mathrm{m})$. The whisker stimulators were oriented in the preferred direction to produce the largest response as determined with the hand probe. Each of the six whisker stimulators were controlled by a computer-programmable Master-8 (AMPI, Jerusalem, Israel) integrated into the MNAP recording system.

Whisker stimulation was delivered according to the following protocols. When six whisker stimulators were used, a single trial contained stimuli for all six whiskers and lasted a total of $14 \mathrm{~s}$. A trial consisted of an initial $2 \mathrm{~s}$ without whisker stimulation, followed by stimulation delivered to each whisker at $2 \mathrm{~s}$ intervals (the order of whisker stimulation was selected randomly). Thus, the first whisker was stimulated $2 \mathrm{~s}$ after the trial began, the second whisker was stimulated $4 \mathrm{~s}$ after the trial started, and so on, so that the sixth (last) whisker stimulus was delivered $12 \mathrm{~s}$ after the start of the trial. Whisker stimuli consisted of 4 stimuli delivered at 2 $\mathrm{Hz}$ or 10 stimuli delivered at 10 or $20 \mathrm{~Hz}$. To afford time, only four stimuli were used at $2 \mathrm{~Hz}$ because little more adaptation is produced at this frequency with additional stimuli. For high-frequency whisker stimulation (e.g., $10 \mathrm{~Hz}$ ) analyses, the last stimulus in the train was used.

Each peristimulus time histogram (PSTH) was created with a minimum of 30 trials and had a $1 \mathrm{~ms}$ bin size. The onset of the $1 \mathrm{~ms}$ whisker deflection coincides with the onset of bin +1 . When all whiskers are stimulated simultaneously, the onset of the whisker stimulation also occurred at $2 \mathrm{~s}$ after the beginning of the trial, and a trial lasted 10 or $5 \mathrm{~s}$. The number of stimuli and frequency used were the same as for the individual whiskers.

During RF stimulation, only two whisker stimulators were used at the same time. A trial lasted $10 \mathrm{~s}$, and the RF stimulation ( $100 \mathrm{~Hz}$ for $1 \mathrm{~s}$ ) was delivered $1 \mathrm{~s}$ after the trial started. The whisker stimulus was delivered $3 \mathrm{~s}$ after the trial started (i.e., $1 \mathrm{~s}$ after the offset of the RF stimulation). The second whisker stimulator was used as a control and stimulated another whisker $5 \mathrm{~ms}$ after the trial started. This response remained stable as the other whisker stimulator was moved between whiskers in a serial manner. When RF stimulation was not delivered (during the quiescent state), a trial had the same duration.

Data analysis. Spontaneous cell firing was computed by counting the number of spikes during the $2 \mathrm{~s}$ period ( $1 \mathrm{~s}$ for RF experiments) at the beginning of each trial and for a minimum of 30 trials. Fast-Fourier 
transforms (FFTs) were calculated to derive a power spectrum of the spontaneous field potential activity. FFTs and PSTHs were calculated using Neuroexplorer (Nex Technologies, Littleton, MA). Additional analyses were performed with Origin software (OriginLab, Northampton, MA). Population data are presented as mean \pm SD. Statistics for comparing population data between quiescent and activated states consisted of paired $t$ tests. Note that each cell is studied in these two different states. Population data analyses compared the PW and the best responding AW.

Histology. At the end of the experiments, the animals were given an overdose of sodium pentobarbital and either perfused through the heart with saline followed by paraformaldehyde (4\%) or the brain was directly extracted and placed in the fixative. The brains were then sectioned in the coronal plane using a vibratome $(80-100 \mu \mathrm{M})$ and processed for Nissl staining. For the cells included in the study, subsequent analysis confirmed the location of electrode tracts within the VPM and within Pr5.

In some cases, recording electrodes were filled with neurobiotin ( $2 \%$ in 1-3 M K-acetate), and attempts were made to label the VPM cells using juxtacellular injections (Pinault, 1996). Stained neurons were visualized through standard avidin-biotin-peroxidase reaction with diaminobenzidine. Briefly, the brains were perfused using $4 \%$ paraformaldehye with $1 \%$ glutaraldehyde. Eighty-micrometer sections were cut using a vibratome. Sections were incubated in $3 \%$ hydrogen peroxide, followed by $0.2 \%$ Triton $\mathrm{X}-100$ and $2 \%$ goat serum. Incubation with $\mathrm{ABC}$ reagent (Vector Laboratories, Burlingame, CA) occurred overnight. The following day, diaminobenzidine was applied to the sections. After color development, sections were mounted and cleared in xylene. Cells were later traced using Neurolucida software (MicroBrightField, Williston, VT).

\section{Results}

\section{Data set}

VPM cells were identified by their short latency to a whisker deflection $(<7 \mathrm{~ms})$ and by tracing the track of the recording electrode in histological sections confirming that the electrode was inside the VPM. In six cases, we attempted juxtacellular labeling of the recorded cells with neurobiotin using previously published protocols (Pinault, 1996). Three of these cases were successful in that one labeled cell was recovered. The labeled cells were found to be located in the VPM in the dorsal part of the nucleus, where the barreloid cores are located. One of those labeled cells, reconstructed using Neurolucida software, is shown in Figure $1 B$. The data shown in the present study is part of a larger data set consisting of 150 well isolated single-unit recordings. Because the amplitude of these spikes is generally in the range of $5-10 \mathrm{mV}$, we presume that the electrode tip is very close to the membrane of the cell. Generally, these recordings are fairly stable so that these cells can be studied for several hours. All of the cells presented here are naïve cells. That is, RF stimulation or carbachol applications were done to one cell per animal.

\section{Effect of activation caused by reduced anesthesia on VPM receptive fields}

Under control conditions, when the field potential activity recorded in the frontal neocortex is stable and no other manipulation has been performed, the responses of VPM cells to controlled whisker stimulation (receptive field of these cells) are quite stable. We used a minimum of 30 whisker stimulation trials to generate a PSTH. When multiple PSTHs are derived over time, they do not vary significantly (data not shown) as long as the anesthetic state is kept constant and the whisker stimulators are kept in the same position. The anesthetic state can be kept constant by monitoring the frontal cortex field potential activity and supplementing it with additional anesthetic as needed. If this supplementation is withheld for some time, the frontal cortex field potential activity may show signs of activation.
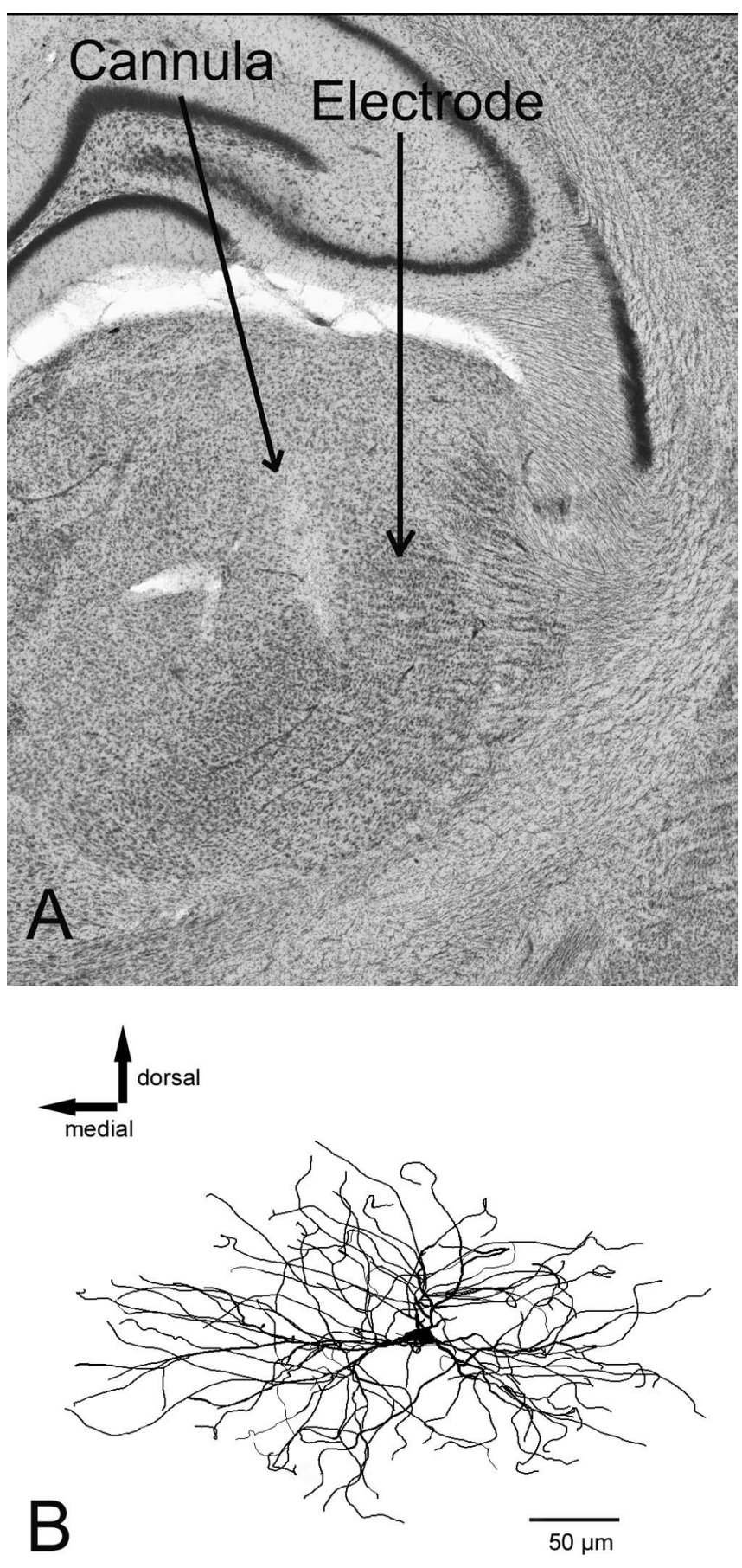

Figure 1. Location of electrodes in the VPM. A, Cresyl violet-stained coronal section shows the positioning of recording electrodes and microdialysis probes in the VPM. Note the tract left by the microdialysis probe entering the VPM. The probe was inserted into the brain at an angle from the midline, and the recording electrode was inserted parallel to the midline into the VPM. The cell shown in $\boldsymbol{B}$ was located in the dorsal portion of the VPM. $\boldsymbol{B}$, Reconstruction of a juxtacellular labeled VPM cell in the coronal plane.

In a number of experiments $(n=5)$, while recording a VPM unit, the animal transitioned between a quiescent state to an activated state. In some cases, this occurred while six independent whisker stimulators were in position, which allowed comparing the responses to deflection of six different whiskers during the two distinct states. Spontaneous activation was evident by the following signs: the recorded VPM cell would enhance its firing rate significantly $(\sim 10$-fold), and the simultaneously recorded 

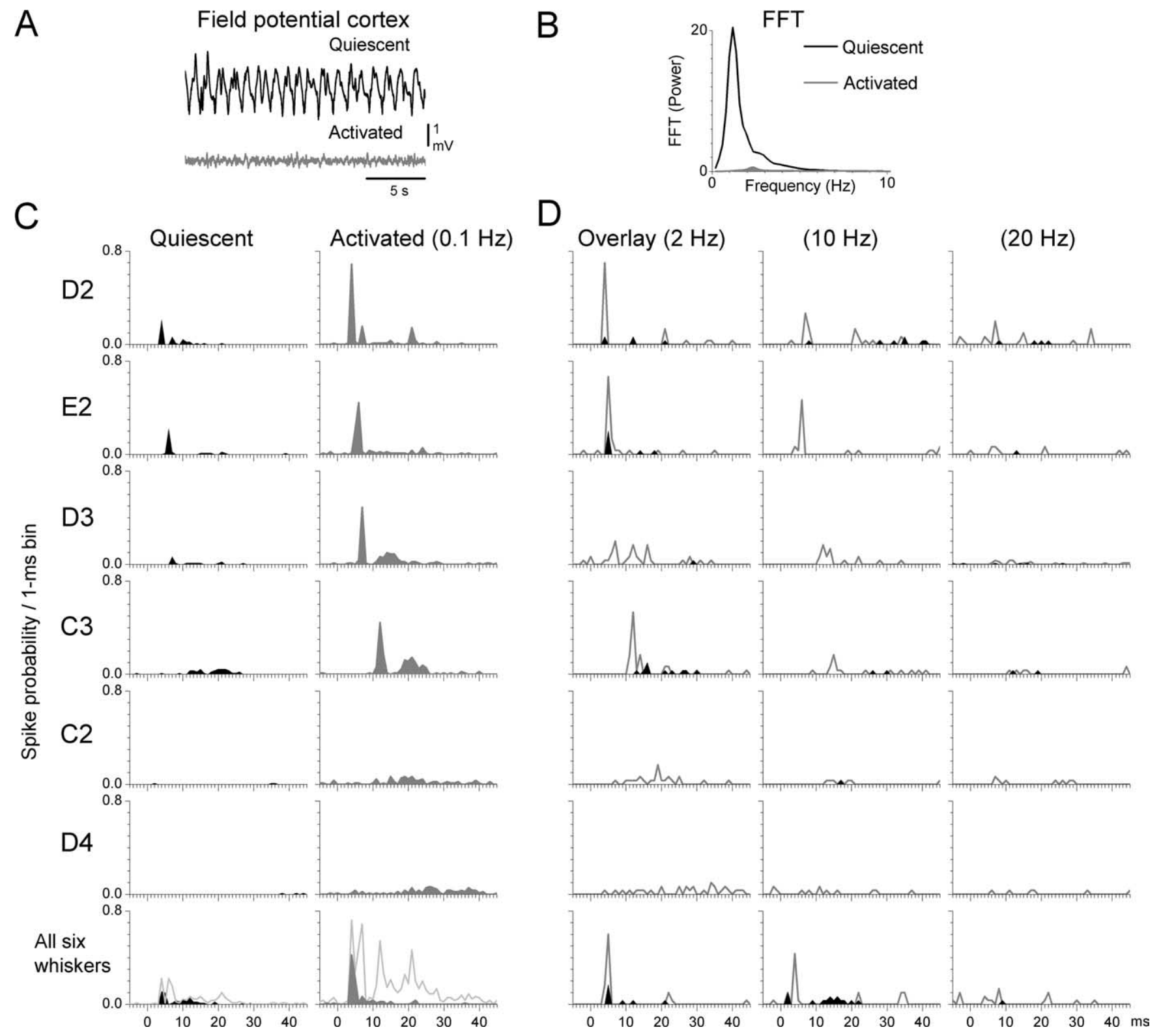

Figure 2. Representative activity of a VPM cell during quiescent and activated states caused by manipulating the level of anesthesia. $A$, Sample field potential activity recorded from the frontal neocortex during quiescent and activated states. $\boldsymbol{B}$, Power-spectrum analysis of the frontal cortex field potential activity derived by calculating FFTs for the quiescent and activated periods during which the PSTHs in Cand D were obtained. C, PSTHs showing responses to stimulation of the PW (D2) and five AWs (E2-D4) during quiescent (black traces) and activated (gray traces) states at 0.1 $\mathrm{Hz}$. The response to simultaneous stimulation of the six whiskers (bottom row) is also shown. The algebraic sum of the responses to individual stimulation of the six whiskers (light gray trace) was overlaid for comparison with the simultaneous stimulation. D, Overlaid PSTHs of whisker stimulation delivered at 2, 10, and $20 \mathrm{~Hz}$ during quiescent (black traces) and activated (gray traces) states.

field potential in the frontal cortex would change from a largeamplitude, slow-wave pattern to a small-amplitude, fast oscillatory pattern. Subsequent application of supplemental anesthetic returned all signs to the quiescent state. It is noteworthy that animals during this activated state were completely unresponsive to a strong tail pinch, displayed no movements, and showed no signs of waking.

An example of a cell displaying a transition between the quiescent and activated states while the whiskers were being stimulated is shown in Figure 2. In all of the figures, gray is used to denote the activated state, and black is used to denote the quiescent state. The spontaneous activity of this cell was measured during a $2 \mathrm{~s}$ time window in the absence of whisker stimulation from the beginning of each trial (measured over all trials). During the quiescent state, the spontaneous firing rate of this cell was 0.3
$\mathrm{Hz}$, whereas during the activated state, the firing rate was $4.5 \mathrm{~Hz}$. Figure $2 \mathrm{~A}$ shows a sample of field potential activity recorded from the frontal cortex during the quiescent and activated states. Note the large-amplitude slow activity during the quiescent state and the low-amplitude fast activity during the activated state. Figure $2 B$ shows a power-spectrum analysis of the frontal cortex field potential activity obtained by calculating an FFT during the quiescent and activated periods during which the PSTHs shown in Figure 2, $C$ and $D$, were obtained. Figure $2 C$ shows PSTHs evoked by single-whisker stimuli at $0.1 \mathrm{~Hz}$ during the quiescent and activated periods. Based on response latency, D2 was considered the PW. During the quiescent state, the cell responded primarily to two of the stimulated whiskers (D2 and E2). However, during activation, the cell responded to stimulation of most of the whiskers, albeit with different latencies and strengths. Similarly, 
Table 1. Population data showing the effect of activation caused by lowering the anesthetic level, by applying electrical stimulation to the reticular formation or by applying carbachol to the thalamus on PW and AW responses and on spontaneous firing in the VPM

\begin{tabular}{|c|c|c|c|c|c|c|}
\hline & $0.1 \mathrm{~Hz}$ & & & $10 \mathrm{~Hz}$ & & \\
\hline & Quiescent & Activated & $t$ & Quiescent & Activated & $t$ \\
\hline Response (spike & & & & & & \\
\hline Anesthesia ( & & & & & & \\
\hline PW & $0.7 \pm 0.1$ & $1.02 \pm 0.05$ & n.s. & $0.16 \pm 0.05$ & $0.66 \pm 0.08$ & * \\
\hline AW & $0.26 \pm 0.04$ & $1.07 \pm 0.15$ & $*$ & $0.1 \pm 0.05$ & $0.61 \pm 0.1$ & * \\
\hline RF stim $(n=$ & & & & & & \\
\hline PW & $0.95 \pm 0.05$ & $1.22 \pm 0.23$ & n.s. & $0.42 \pm 0.23$ & $0.88 \pm 0.22$ & * \\
\hline AW & $0.4 \pm 0.12$ & $1.11 \pm 0.25$ & * & $0.12 \pm 0.07$ & $0.58 \pm 0.32$ & $* *$ \\
\hline Carbachol ( $n$ & & & & & & \\
\hline PW & $0.79 \pm 0.11$ & $1.03 \pm 0.1$ & n.s. & $0.26 \pm 0.16$ & $1.06 \pm 0.23$ & * \\
\hline AW & $0.28 \pm 0.18$ & $0.99 \pm 0.13$ & $*$ & $0.11 \pm 0.1$ & $0.81 \pm 0.13$ & * \\
\hline AW/PW ratio & & & & & & \\
\hline Anesthesia & $0.39 \pm 0.07$ & $1.03 \pm 0.09$ & * & $0.57 \pm 0.26$ & $0.89 \pm 0.12$ & n.s. \\
\hline RF stim & $0.42 \pm 0.12$ & $0.94 \pm 0.23$ & * & $0.42 \pm 0.24$ & $0.58 \pm 0.23$ & n.s. \\
\hline Carbachol & $0.35 \pm 0.19$ & $0.96 \pm 0.09$ & * & $0.22 \pm 0.15$ & $0.75 \pm 0.11$ & $*$ \\
\hline Spontaneous fir & & & & & & \\
\hline Anesthesia & $0.28 \pm 0.2$ & $2.9 \pm 1.8$ & * & & & \\
\hline RF stim & $0.4 \pm 0.3$ & $7.5 \pm 6$ & * & & & \\
\hline Carbachol & $0.12 \pm 0.1$ & $5.6 \pm 5$ & * & & & \\
\hline
\end{tabular}

Three parameters are displayed: responses, AW/PW ratio of the responses, and spontaneous firing rate. Responses were measured by summing the spikes evoked during a $20 \mathrm{~ms}$ time window starting $3 \mathrm{~ms}$ after the whisker stimulus. Spontaneous firing was measured during a 1 or $2 \mathrm{~s}$ time window before the whisker stimulation during each trial. Data are mean $\pm S D$. $t$ test comparisons are between the quiescent and activated states $\left({ }^{*} p<0.01 ;{ }^{* *} p<0.05\right)$. n.S. Nonsignificant; stim, stimulation.

Figure $2 \mathrm{D}$ overlays PSTHs corresponding to the 10 th whisker stimulus of trains delivered at different frequencies $(2,10$, and 20 $\mathrm{Hz}$ ) during the quiescent and activated periods. During the quiescent state, there were practically no responses to whisker stimulation at 10 and $20 \mathrm{~Hz}$, whereas during the activated state, responses (albeit reduced) were present for most whiskers at $10 \mathrm{~Hz}$ and for the PW at $20 \mathrm{~Hz}$. Thus, whisker responses at frequencies $>2 \mathrm{~Hz}$ were more strongly suppressed during the quiescent state than the activated state, as shown previously during simultaneous stimulation of several whiskers (Castro-Alamancos, 2002b). In this cell, subsequent application of a supplemental anesthetic dose completely reversed all of the effects of activation. Thus, the PSTHs, field potential activity and spontaneous firing rate returned to the quiescent state (data not shown). This cell was also studied during application of carbachol to the VPM, as detailed below.

In addition to testing the responses of individual whiskers, we also studied the effect of simultaneously stimulating all six whiskers (Fig. 2C,D, bottom). The PSTHs revealed two interesting effects of simultaneous whisker stimulation. First, the response obtained by stimulating all six whiskers together was similar to the response of the PW alone (albeit somewhat suppressed). Second, there was an abolishment of the long-latency responses that would be evoked by the AWs. This is apparent if the response evoked by stimulating all six whiskers simultaneously is compared with the algebraic sum of the responses to the six individual whiskers. The sum is overlaid (light gray trace) in the bottom panels of Figure $2 C$. The result indicates that during simultaneous stimulation of all six whiskers, the long-latency responses characteristic of AWs are absent. This effect was particularly noticeable during the activated state, the state during which the responses to stimulation of individual AWs are stronger. We will discuss this nonlinear summation or suppression of AWs responses resulting from simultaneous multiwhisker stimulation below (see Single-whisker versus multiwhisker responses).

Population data (Table 1) were obtained from five cells that were observed to transition spontaneously between a quiescent state and an activated state. For these cells, the average firing rate increased 10-fold during the activated period compared with the quiescent period (Table 1). A "response" for each whisker stimulus was calculated by summing the spikes during a $20 \mathrm{~ms}$ time window starting $3 \mathrm{~ms}$ after the whisker deflection. In these analyses, the PW and one AW were compared. We found that for low-frequency whisker stimulation $(0.1 \mathrm{~Hz})$, the response to the PW was slightly enhanced during activation compared with the quiescent state, but this change did not reach statistical significance (Table 1). In contrast, the response to the AW was significantly enhanced during the activated state ( $t$ test; $p<0.01)$ compared with the quiescent state. For high-frequency whisker stimulation $(10 \mathrm{~Hz})$, the responses to both the PW and AW were significantly enhanced ( $t$ test; $p<0.01$ ) compared with the quiescent state. These results indicate that for low-frequency whisker stimulation, activation increases more the AW responses, so that PW and AW responses are similar during activation. For highfrequency whisker stimulation, the responses of PWs and AWs are both suppressed compared with low-frequency responses. Activation increases both of these responses similarly. Consequently, during activation, the number of spikes evoked during a $20 \mathrm{~ms}$ time window after the whisker deflection is similar for PWs and AWs. Note that, by definition, the PW responses have a shorter latency than the AW responses.

An indication of the relative strength of PW over AW responses is provided by calculating a ratio between them (Table 1). An AW/PW ratio of 1 indicates that there is no difference between these responses. For low-frequency whisker stimulation $(0.1 \mathrm{~Hz})$ in the quiescent state, the ratio is 0.39 , indicating that the PW produces a much stronger response than the AW. However, during the activated state, this ratio changes significantly $(p<$ 0.01 ) and reaches a value of $\sim 1$, indicating that there is no difference between the PW and AW responses during activation. For high-frequency whisker stimulation $(10 \mathrm{~Hz})$ in the quiescent state, the ratio is 0.57 , indicating that there is a tendency for the PW to produce a stronger response than the AW. During the activated state, this ratio changed to 0.9 , indicating little differences between PW and AW responses. These results show that activation increases the receptive fields of VPM neurons differ- 


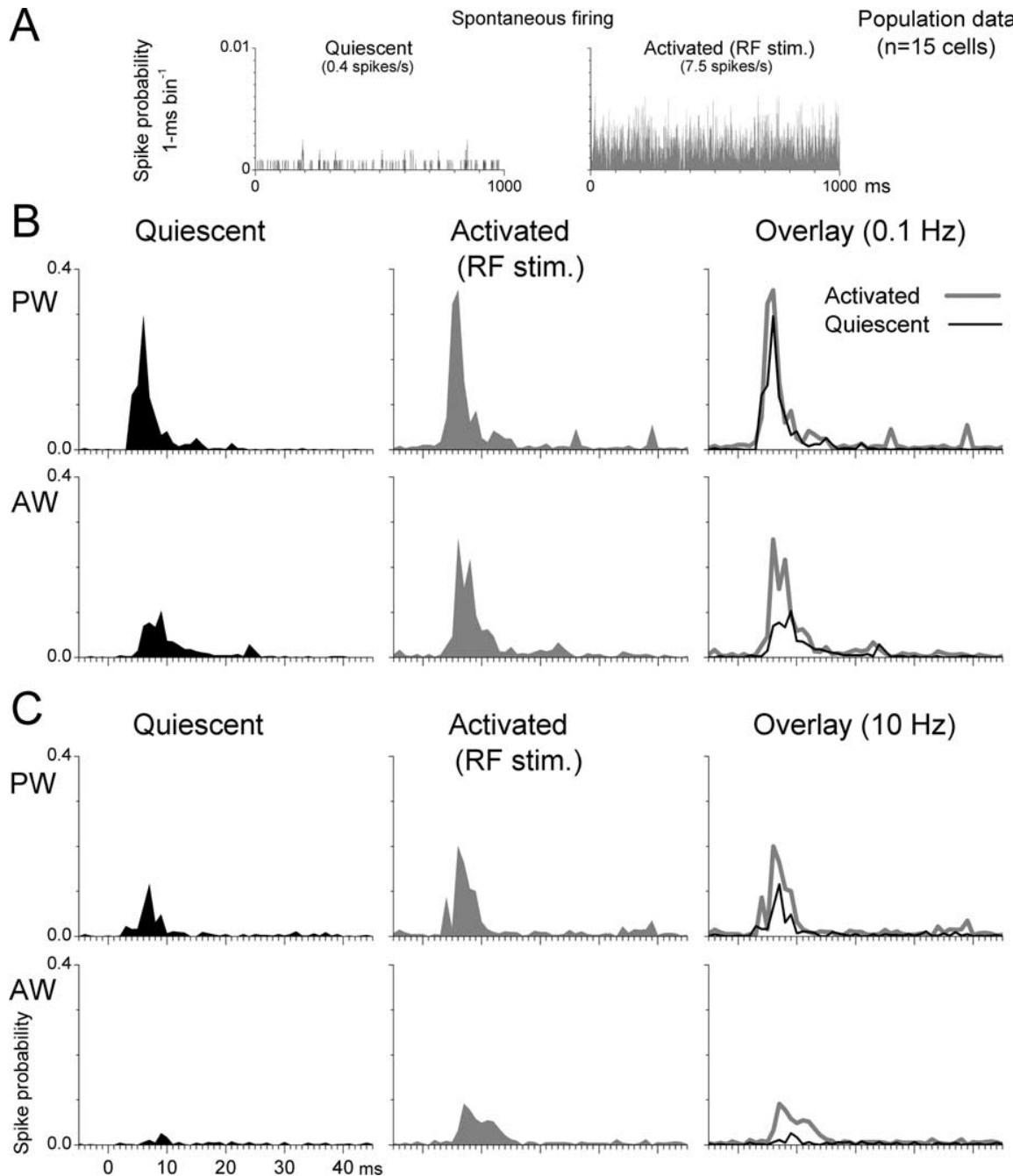

Figure 3. Population PSTHs during quiescent and activated states caused by RF stimulation. $\boldsymbol{A}$, Spontaneous activity of the VPM cells $(n=15)$ measured during a 1 s time window in the absence of whisker stimulation during quiescent and activated states. During RF stimulation trials, this window corresponds to the period between the offset of the RF stimulation and the onset of the whisker stimulation. During control (quiescent) trials, this period corresponds to $1 \mathrm{~s}$ before the whisker stimulus. $\boldsymbol{B}$, Average PSTHs of low-frequency $(0.1 \mathrm{~Hz})$ PW and AW responses during quiescent (black traces) and activated (gray traces) states. $\boldsymbol{C}$, Average PSTHs of high-frequency $(10 \mathrm{~Hz})$ PW and AW responses during quiescent and activated states. stim., Stimulation.

the RF stimulation (see Materials and Methods). The firing rate of the cell was measured during the $1 \mathrm{~s}$ period between the offset of the RF stimulation (if present) and the onset of the whisker stimulation for a minimum of 30 trials.

An example of the responses of a VPM cell for which seven whiskers were stimulated serially before and during activation caused by RF stimulation is shown in supplemental Figure 1 (available at www. jneurosci.org as supplemental material). The spontaneous firing rate of this cell was $0.7 \mathrm{~Hz}$ during the quiescent state and $9 \mathrm{~Hz}$ during the activated state caused by RF stimulation. Based on response latency, C6 was the PW. During the quiescent state, when the spontaneous activity of the cell was low, the cell responded primarily to whiskers $\mathrm{C} 6$ and $\mathrm{C} 7$ and, to a lesser extent, to whiskers C5 and D6. However, during activation caused by RF stimulation, the cell responded to stimulation of most of the seven whiskers. Whisker stimulation at $10 \mathrm{~Hz}$ strongly suppressed the responses to all whiskers during quiescent states, whereas during activation caused by RF stimulation, the responses of the PW and some AWs were noticeable.

Similar results were obtained from all of the cells tested with RF stimulation. PSTHs derived from a population of VPM cells $(n=15)$ that were subjected to RF stimulation are shown in Figure 3 for both low-frequency $(0.1 \mathrm{~Hz})$ and highfrequency $(10 \mathrm{~Hz})$ whisker stimulation. The PW and one AW are compared. Table 1 quantifies these responses. For lowfrequency whisker stimulation $(0.1 \mathrm{~Hz})$, the response to the PW was slightly enhanced by activation, but this change did not reach statistical significance. In contrast, the response to the AW was signifi-

ently for low- and high-frequency whisker stimuli. For lowfrequency whisker stimuli, activation enhances more the responses to AWs. For high-frequency whisker stimuli, activation increases both the responses to PWs and AWs, which are both suppressed by frequency.

\section{Effect of RF stimulation on VPM receptive fields}

The previous results indicate that activation produced by changes in the level of anesthesia cause significant changes in the receptive fields of VPM cells. The next step was to test whether this could also be induced by RF stimulation while keeping the level of anesthesia constant. RF stimulation produces activation that affects the temporal responses of VPM and barrel cortex cells (Castro-Alamancos, 2002b, 2004a; Castro-Alamancos and Oldford, 2002). Thus, we tested the effect of RF stimulation on the receptive fields of VPM neurons. In these experiments, each whisker was stimulated in a serial manner. This assured that for every trial, each whisker stimulus was delivered at the same time in relation to the RF stimulation $(100 \mathrm{~Hz}$ for $1 \mathrm{~s})$. The data shown corresponds to stimulation of each whisker $1 \mathrm{~s}$ after the offset of cantly enhanced by activation ( $t$ test; $p<0.01$ ). For highfrequency whisker stimulation $(10 \mathrm{~Hz})$, the responses to the PW and AW were suppressed compared with low-frequency responses $(0.1 \mathrm{~Hz})$. However, during activation caused by RF stimulation, the responses to the PW and AW were both enhanced significantly ( $t$ test; $p<0.01$ and $p<0.05$, respectively) compared with the quiescent state. These results indicate that for low-frequency whisker stimulation, activation mostly increases the AW responses, so that $\mathrm{PW}$ and AW responses are similar during activation. For high-frequency whisker stimulation, the responses of PWs and AWs are suppressed compared with lowfrequency whisker responses, and activation increases both of these responses significantly. These results resemble those obtained when activation was caused by changing the anesthetic level.

The ratio between the AW and PW responses for VPM cells subjected to RF stimulation, which gives an indication of their relative strengths, is shown in Table 1 . For low-frequency whisker stimulation $(0.1 \mathrm{~Hz})$ in the quiescent state, the ratio is 0.42 , indicating that the PW produces a much stronger response than the 
AW. However, during the activated state, this ratio changes significantly $(p<0.01)$ and reaches a value of $\sim 1$, indicating that there is no difference between the PW and the AW responses during activation. For high-frequency whisker stimulation (10 $\mathrm{Hz}$ ) in the quiescent state, the ratio is 0.42 , indicating that the PW has a stronger response than the AW. During the activated state, the ratio changes to 0.58 , but this effect did not reach statistical significance. Thus, the PW remained preponderant during high-frequency stimulation in the activated state. This effect appears to reflect the fact that during activated states, many AWs are able to drive VPM cells only at low frequencies (for example, see whiskers D3 and C3 in Fig. 2 and whiskers C7 and C5 in supplemental Fig. 1, available at www.

jneurosci.org as supplemental material), whereas others can also drive them at higher frequencies.

\section{Effect of RF stimulation on Pr5 receptive fields}

The previous results indicate that activation produced by changes in the level of anesthesia or by RF stimulation produce significant changes in the receptive fields of VPM cells. Both of these procedures cause generalized changes in activation; activation can be measured throughout the forebrain during these conditions. It is also possible that changes may be happening at the level of the brainstem in $\operatorname{Pr} 5$ that could account for these results. Pr5 cells in lightly anesthetized animals have large receptive fields that can explain the large receptive fields observed in the VPM during activation (Minnery and Simons, 2003; Minnery et al., 2003). Thus, if RF stimulation was enlarging the receptive fields of Pr5 cells, then VPM cells would simply be reflecting such an enlargement. Alternatively, during quiescent states, the receptive fields of $\operatorname{Pr} 5$ cells may already be large, and the thalamus would suppress them. Hence, activation would cause enlargement of VPM receptive fields by a direct thalamic effect. To test these possibilities, we recorded from Pr5 cells during quiescent states and during activation caused by RF stimulation.

In a first set of experiments, we recorded simultaneously from Pr5 cells and from VPM cells. Figure $4 A$ shows the effect of RF stimulation on the spontaneous firing of $\operatorname{Pr} 5$ cells $(n=18)$ and on a population of VPM cells ( $n=7$ pairs) that were simultaneously recorded with a group of the Pr5 cells. RF stimulation had no significant effect on the population of $\operatorname{Pr} 5$ cells $(3.3 \pm 5$ vs $3.3 \pm$ $5 \mathrm{~Hz}$; quiescent vs activated), whereas the simultaneously recorded VPM cells showed a significant increase in firing rate $(0.8 \pm 0.7$ vs $10.1 \pm 7 \mathrm{~Hz})$. The simultaneous recording from $\operatorname{Pr} 5$ and the VPM assured that the RF stimulation was effectively activating the thalamus. In a second set of experiments, we recorded from Pr5 cells that project to the VPM as determined using antidromic invasion tests performed with a stimulating electrode placed in the VPM [antidromic spike latency for this population was $0.9 \pm 0.3 \mathrm{~ms}(n=8)$, similar to that in the study by Minnery and Simons (2003)]. RF stimulation had no significant effect on the spontaneous firing rate of identified VPMprojecting Pr5 cells (similar to Fig. $4 A$ ). Figure $4 B$ shows the effect of RF stimulation on PW and AW responses from the population of $\operatorname{Pr} 5$ cells that were not identified using antidromic procedures ( $n=18$; first set of experiments) and from the population of Pr5 cells that were found to project to the VPM $(n=8$; second set of experiments). Because these two populations of cells did not differ significantly, we combined the data into the same PSTHs $(n=26)$ shown in Figure $4 B$. In none of the cells did the RF stimulation cause a significant increase in the PW or AW responses at either $0.1 \mathrm{~Hz}$ (Fig. $4 B$ ) or $10 \mathrm{~Hz}$ (data not shown). These results show that RF stimulation does not affect the receptive fields of VPM-projecting Pr5 cells.

\section{Effect of thalamic activation on VPM receptive fields}

Activation produced by changes in the level of anesthesia or by RF stimulation cause significant changes in the receptive fields of VPM cells, and this effect cannot be explained by a change at the level of Pr5. Both of these procedures cause generalized changes in activation throughout the forebrain. Thus, we were interested in testing whether a more selective activation of the thalamus would also result in significant changes in the receptive fields of VPM cells. To produce thalamic activation, we infused a cholinergic agonist (carbachol; $500 \mu \mathrm{M}$ ) into the VPM using microdialysis, as described previously (Castro-Alamancos, 2002b). In these experiments, up to six whisker stimulators were used simultaneously to map the responses of the PW and several AWs. 

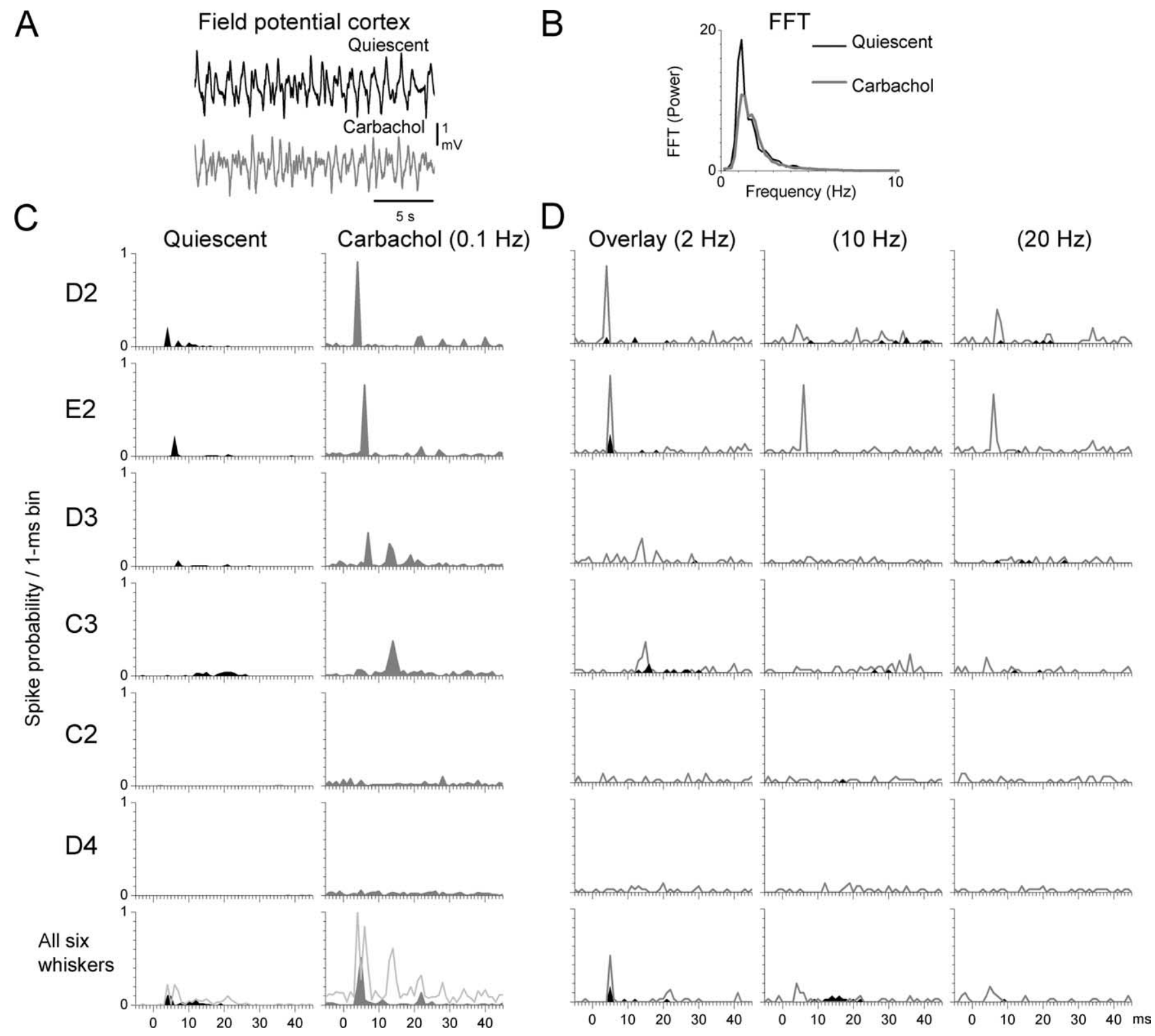

Figure 5. Representative activity of a VPM cell during thalamic activation caused by application of carbachol in the VPM. A, Sample field potential activity recorded from the frontal neocortex during quiescent and thalamic activation states caused by carbachol application in the VPM. B, Power-spectrum analysis of the frontal cortex field potential activity derived by calculating FFTs for the quiescent and thalamic activated periods during which the PSTHs in $\boldsymbol{C}$ and $\boldsymbol{D}$ were obtained. C, PSTHs showing responses to stimulation of the PW (D2) and five AWs (E2-D4) during quiescent (black traces) and activated (gray traces) states. The response to simultaneous stimulation of the six whiskers (bottom row) is also shown. The algebraic sum of the responses to individual stimulation of the six whiskers (light gray trace) was overlaid for comparison with the simultaneous stimulation. D, Overlaid PSTHs of whisker stimulation delivered at 2, 10, and 20 Hz during quiescent (black traces) and thalamic activated (gray traces) states.

An example of the effects of carbachol on a typical VPM cell is shown in Figure 5. This example was chosen because it corresponds to the same cell shown in Figure 2, which previously transitioned between a quiescent and an activated state as the anesthetic supplementation was withheld. This allows comparing the effects of generalized forebrain activation with a more selective thalamic activation in the same cell. During the quiescent state, the spontaneous firing rate of the cell was $0.3 \mathrm{~Hz}$, whereas during carbachol, the firing rate was $20.2 \mathrm{~Hz}$. Figure $5, A$ and $B$, shows a sample of field potential activity recorded from the frontal cortex during the quiescent state and during carbachol, and a related FFT analysis. Note the large-amplitude slow activity during the quiescent state and also during the application of carba- chol into the thalamus. This shows that although carbachol activated the thalamus, it did not produce a generalized activation of the forebrain. Thus, the level of anesthesia remained constant. Figure $5 C$ shows PSTHs evoked by stimulation of six individual whiskers at $0.1 \mathrm{~Hz}$ during the quiescent and carbachol periods. During the quiescent state, when the spontaneous activity of the cell was low, the cell responded primarily to two of the stimulated whiskers (D2 and E2). However, during carbachol, the cell responded to stimulation of four of the whiskers, albeit with different latencies and strengths. Figure $5 D$ overlays PSTHs of responses at different frequencies $(2,10$, and $20 \mathrm{~Hz})$. During the quiescent state, there were practically no responses to whisker stimulation at 10 and $20 \mathrm{~Hz}$, whereas during the carbachol pe- 
riod, responses (albeit reduced) were present for most whiskers at $2 \mathrm{~Hz}$ and for whiskers D2 and E2 at $20 \mathrm{~Hz}$.

We also tested the effect of simultaneously stimulating all six whiskers (Fig. 5C,D, bottom). As shown above for activation caused by lowering the anesthetic (Fig. 2), the response produced by stimulating all whiskers simultaneously resembled the PW response, and the long-latency response components of AWs were absent. The algebraic sum of the responses to the six individual whiskers is overlaid (light gray trace) in the bottom panels of Figure $5 \mathrm{C}$. Thus, the effects of spontaneous activation (Fig. 2) and carbachol into the VPM (Fig. 5) are quite similar in the same cell.

The VPM consists of barreloids that contain a core region in the mediodorsal part and a tail region in the ventrolateral part. The core region receives inputs from $\operatorname{Pr} 5$, whereas the tail region receives inputs from the interpolaris spinal trigeminal nucleus (Williams et al., 1994; Veinante et al., 2000). Thus, it is possible that some of the cells we recorded in the VPM were located in the tail region and received input from interpolaris. These cells may have fairly large receptive fields because interpolaris cells have quite large receptive fields (Woolston et al., 1982; Jacquin et al., 1986). It is important to note that all of the cells we recorded and tested with carbachol enlarged their receptive fields. Hence, it is unlikely that there is a difference in this respect between cells in the core region and those in the tail region of barreloids in the VPM, unless we completely missed tail region cells. One putative tail region cell, based on its large receptive field during quiescent states, is shown in supplemental Figure 2 (available at www. jneurosci.org as supplemental material). These cells were rare in our total population, and only two cells were subjected to application of carbachol. As shown, this cell enhanced its responses to AWs and consequently enlarged its receptive field in response to selective thalamic activation.

An additional issue relates to the muscarinic receptor mediation of the cholinergic effects produced by carbachol application. To test whether carbachol was indeed acting on muscarinic receptors, we applied atropine into the thalamus via microdialysis ( $1 \mathrm{~mm}$ ) immediately after carbachol ( $n=5$ cells). In every cell tested, atropine resulted in an immediate and complete reversal of the effects of carbachol. An example of the effects of atropine in one cell is shown in supplemental Figure 2 (available at www. jneurosci.org as supplemental material).

Similar results were obtained from all of the cells tested with carbachol. PSTHs derived from a population of VPM cells $(n=$ 10) that were subjected to application of carbachol (data not shown) are very similar to those shown in Figure 3 during RF stimulation. Carbachol markedly increased the spontaneous firing rate of VPM cells (Table 1). The responses from the PSTHs for VPM cells subjected to carbachol application are quantified in Table 1. For low-frequency whisker stimulation $(0.1 \mathrm{~Hz})$, the response to the PW was slightly enhanced by carbachol, but this change did not reach statistical significance. In contrast, the response to the AW was significantly enhanced during the application of carbachol into the VPM $(t$ test; $p<0.01)$. For highfrequency whisker stimulation $(10 \mathrm{~Hz})$, the responses to the PW and AW were suppressed compared with low-frequency responses $(0.1 \mathrm{~Hz})$. However, during carbachol, both of these responses were significantly enhanced ( $t$ test; $p<0.01$ ). These results indicate that for low-frequency whisker stimulation, carbachol in the VPM selectively increases the AW responses, so that PW and AW responses are similar. For high-frequency whisker stimulation, the responses of both the PW and AW increase significantly.

The ratio between the AW response and the PW response for
VPM cells tested with carbachol is shown in Table 1. For lowfrequency whisker stimulation $(0.1 \mathrm{~Hz})$ in the quiescent state, the ratio is 0.35 , indicating that the $\mathrm{PW}$ produces a much stronger response than the AW. However, during carbachol, this ratio changes significantly $(p<0.01)$ and reaches a value of $\sim 1(0.96)$, indicating that there is no difference between the PW and AW responses. For high-frequency whisker stimulation $(10 \mathrm{~Hz})$ in the quiescent state, the ratio is 0.22 . During the application of carbachol, the ratio is significantly ( $p<0.01$ ) changed to 0.75 , indicating a smaller difference exists between the PW and AW during carbachol. Thus, PWs remain somewhat preponderant during high-frequency stimulation.

For the VPM cells subjected to carbachol $(n=10)$, we also measured the absolute response latency from stimulus onset to study how this is affected by thalamic activation. The onset latency for PW responses during low-frequency whisker stimulation $(0.1 \mathrm{~Hz})$ is $4.67 \pm 1 \mathrm{~ms}$, and this value was reduced by carbachol to $4.23 \pm 0.7 \mathrm{~ms}$ (this difference was barely statistically significant; $t$ test; $p=0.05$ ). During high-frequency whisker stimulation $(10 \mathrm{~Hz})$, the onset latency of PW responses was much larger $(7.95 \pm 3.8 \mathrm{~ms})$, but it was sharply $(p<0.01)$ shortened by carbachol ( $4.5 \pm 1 \mathrm{~ms})$. The onset latency of AW responses during low-frequency whisker stimulation $(0.1 \mathrm{~Hz})$ was longer $(6.4 \pm 2 \mathrm{~ms})$ than for PW responses and was significantly $(p<$ $0.05)$ reduced by carbachol $(6.1 \pm 1.7 \mathrm{~ms})$. Finally, during highfrequency stimulation $(10 \mathrm{~Hz})$, the onset latency of AW responses $(9.3 \pm 2.9 \mathrm{~ms})$ was also significantly $(p<0.01)$ reduced by carbachol ( $6.5 \pm 1.3 \mathrm{~ms})$. Thus, thalamic activation significantly affects the timing of whisker-evoked responses by reducing the onset latency of both PW and AW responses. This reduction is particularly marked for high-frequency sensory stimuli.

The results of a selective thalamic activation caused by application of carbachol closely resemble those observed during generalized forebrain activation, indicating that during quiescent states, AW responses are suppressed at the level of the thalamus and that thalamic activation enhances these responses, resulting in a larger VPM receptive field.

\section{Single-whisker versus multiwhisker responses}

We found that during activated states, VPM cells are responsive to several AWs in addition to the PW. However, when all of these whiskers are stimulated together, the response obtained resembles the PW response because the longer-latency responses typical of the AWs are absent. Figure $6 A$ shows population data $(n=$ 6) of single-whisker versus multiwhisker stimulation $(0.1 \mathrm{~Hz})$. Shown are either PSTHs obtained by stimulating simultaneously six whiskers (the PW and five AWs) or one PSTH derived from the algebraic sum of the responses to stimulating each of the six whiskers individually. Note that during either the quiescent state or thalamic activation caused by carbachol, the response to stimulating all six whiskers simultaneously is much smaller than the algebraic sum of the individual responses. When these responses are overlaid, it is apparent that only the shortest latency response is present when all six whiskers are stimulated simultaneously. Thus, multiwhisker stimulation causes a nonlinear summation of the responses to the individual whiskers during both quiescent and activated states.

To compare more directly PW responses and AW responses, we summed the responses to the five AWs and overlaid them with the PW response in Figure $6 \mathrm{~B}$. The sum of the responses of the five AWs shows that these whiskers evoke robust responses individually, especially during activated states. However, most noticeable from these PSTHs is that the response to the PW resem- 
A

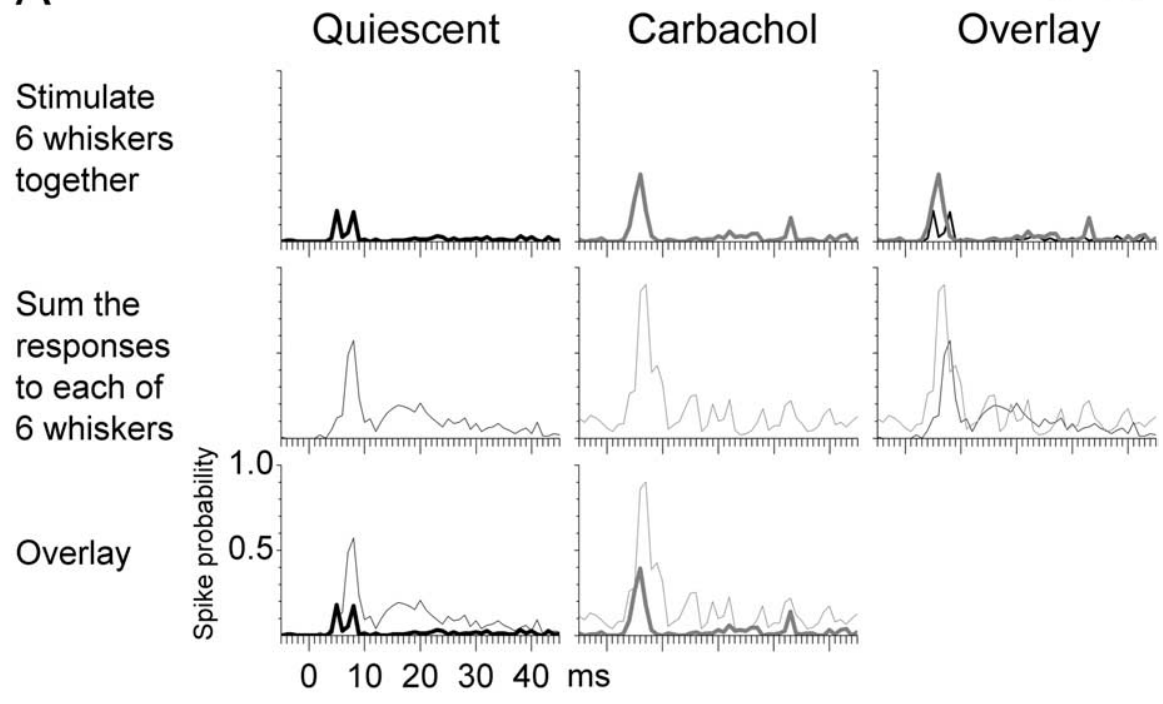

B

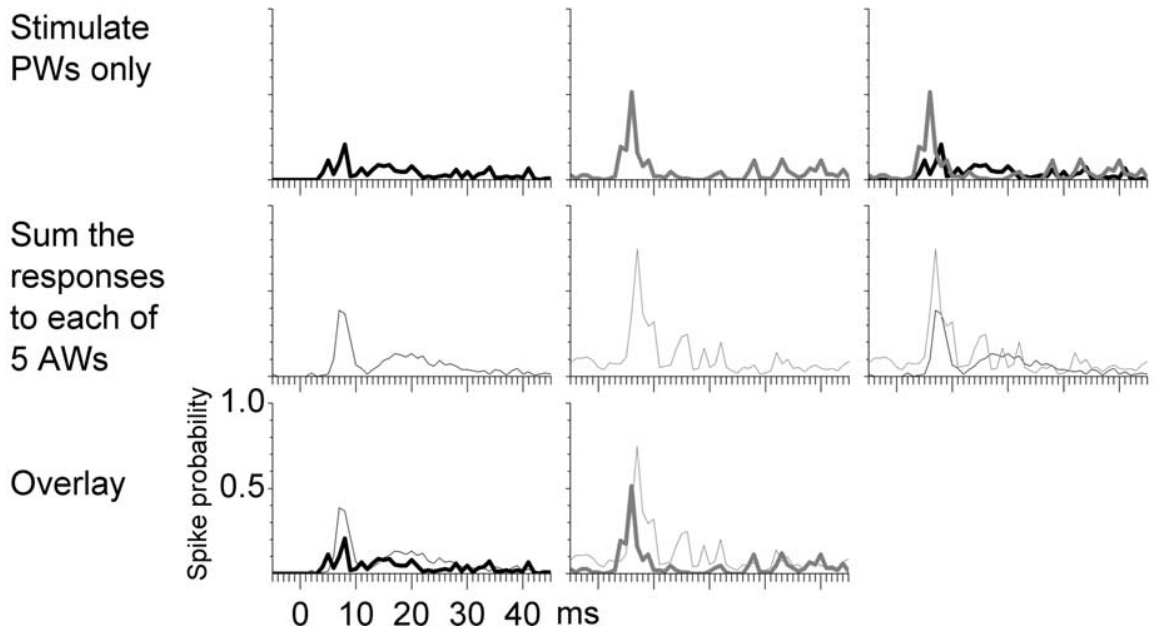

Figure 6. Population PSTHs of multiwhisker responses. $A$, Population PSTH ( $n=6)$ of responses evoked by simultaneously stimulating six whiskers, including the PW and five AWs (top row) and PSTHs consisting of the algebraic sum of the responses to each of these six whiskers stimulated alone (middle row) during quiescent states and during thalamic activation caused by application of carbachol in the VPM. The right column and bottom row overlay the PSTHs of responses shown in the vertically or horizontally aligned panels for comparison. $\boldsymbol{B}$, Population PSTH $(n=6)$ of responses evoked by stimulating the PW alone (top row) or PSTHs consisting of the algebraic sum of the responses to each of the five AWs (middle row) during quiescent states and during thalamic activation caused by application of carbachol in the VPM. The right column and bottom row overlay the PSTHs of responses shown in the vertically or horizontally aligned panels for comparison. PSTHs display spike probability per $1 \mathrm{~ms}$ bin. bles very closely the response to stimulating all six whiskers together. This is compared in Figure $7 A$, in which the responses to the PW alone and the responses to all six whiskers stimulated simultaneously are overlaid. Note that the responses to the PW alone resemble very closely the response to simultaneously stimulating all six whiskers, as if the AWs had not been stimulated. Figure $7 B$ quantifies these responses and shows that although the responses to all six whiskers were slightly suppressed compared with the PW response, this suppression did not reach statistical significance. Moreover, this suppression appears to be mostly caused by a reduction in longer-latency spikes during simultaneous stimulation of the six whiskers. Hence, simultaneous stimulation of PW and five AWs produces responses that closely re- semble the PW response both during quiescent and activated states and for either low- or high-frequency whisker stimulation.

\section{Discussion}

Excitatory receptive fields of VPM cells consist of an excitatory center, the PW, and an excitatory surround, the AWs. The present study shows that activation enlarges the excitatory surround of VPM cells. Thus, for low-frequency sensory inputs, during quiescent states, the response to the PW (receptive field excitatory center) is stronger than the response to AWs (receptive field excitatory surround), but during activation, there is an enhancement of the response to AWs, which can reach response levels similar to the PW. For high-frequency sensory inputs, during quiescent states, both PW and AW responses are depressed because of the lowpass filtering at the lemniscal pathway. However, during activation, there is a significant increase in both PW and AW responses so that they become similar, but PW responses are generally stronger than AW responses at high frequencies.

These effects of activation are caused by either generalized forebrain activation or a more selective thalamic activation attributable to application of carbachol in the thalamus, indicating that the thalamus is the site where most of the receptive field transformation is occurring. Moreover, the effects of activation on VPM receptive fields cannot be explained by changes in Pr5. Thus, during quiescent states, thalamic mechanisms suppress AW responses (the excitatory surround), whereas during activated states, thalamic mechanisms enhance AW responses.

Interestingly, although there is a strong enhancement of the responses to AWs during activation, when the PW and AWs are stimulated simultaneously, the response to the PW prevails. In fact, all response components other than the shortlatency PW response are abolished during multiwhisker stimulation. Hence, there is a nonlinear summation of the responses to all stimulated whiskers. In conclusion, despite the enlargement in excitatory receptive field size during activated states, when the entire receptive field is stimulated simultaneously, it shrinks to the PW (i.e., to the center of the receptive field). Therefore, VPM cells represent only the center of the receptive field when the center and surround are stimulated simultaneously.

An important consideration is whether the effects produced by thalamic activation are mediated solely by cholinergic (muscarinic) receptor activation; we do not believe this is the case. Acetylcholine is an important neuromodulator of sensory and motor systems (Vanderwolf, 1988; Oldford and Castro- 
A PW (thin trace) versus ALL six whiskers (thick trace)
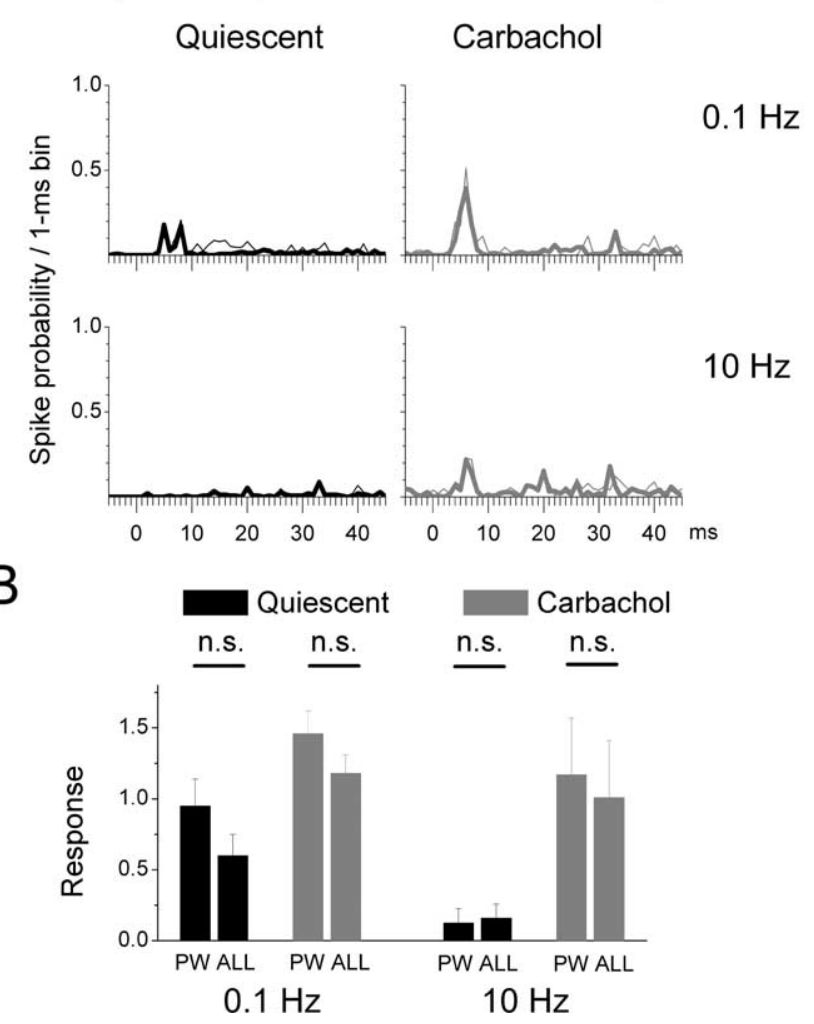

Figure 7. Comparison of responses evoked by the $P W$ alone and responses evoked by all six whiskers stimulated together. $\boldsymbol{A}$, Population PSTH $(n=6)$ of responses evoked by stimulating the PW alone (thin traces) or all six whiskers simultaneously, including the PW (thick traces). Different panels correspond to responses during quiescent and thalamic activation states caused by application of carbachol, during low-frequency $(0.1 \mathrm{~Hz})$ and high-frequency $(10 \mathrm{~Hz})$ whisker stimulation. $\boldsymbol{B}$, Comparison of responses evoked by the PW and all six whiskers during the different conditions. Responses were measured by summing the spikes evoked during a 20 ms time window starting $3 \mathrm{~ms}$ after the whisker stimulus (non-paired $t$ test). For $10 \mathrm{~Hz}$ stimulation, the last response in a 10 stimuli train was used. Error bars represent SD. n.s., Nonsignificant.

Alamancos, 2003; Berg et al., 2005). However, it is unlikely that acetylcholine is the only neuromodulator that can enlarge thalamocortical receptive fields. In fact, preliminary evidence indicates that similar effects are produced by other neuromodulators applied to the thalamus, although each neuromodulator seems to produce distinguishing effects.

Another consideration regarding sensory responses of VPM cells during quiescent states relates to the incidence of bursts evoked by sensory stimulation. Bursts can be unequivocally identified in our single-unit recordings, and they are extremely rare to nonexistent in both the quiescent and the activated states as defined in the present study. During deeper levels of anesthesia caused by much larger doses of urethane than those used here, we find that cells can begin bursting spontaneously, but in this state, the responses to whisker deflections become very unreliable. Certainly, the incidence of bursting is higher during barbiturate anesthesia.

\section{Emergence of AW responses during activation}

Under light anesthesia, VPM cells respond to deflections of multiple whiskers (Simons and Carvell, 1989; Armstrong-James and Callahan, 1991; Diamond et al., 1992; Nicolelis and Chapin, 1994; Friedberg et al., 1999; Brecht and Sakmann, 2002; Minnery et al.,
2003). Recent studies in vivo and in slices indicate that VPM neurons are contacted by a few (one to three) lemniscal fibers, each of them producing fast-rising, large-amplitude EPSPs (Castro-Alamancos, 2002a,b; Deschenes et al., 2003). It is believed that PW responses arise from this innervation. Several possibilities have been considered to explain the origin of the AW responses in VPM neurons. These include corticothalamic feedback, extra-barreloid dendrites that sample inputs from adjacent barreloids, and a direct projection from the spinal trigeminal nucleus [for a discussion, see Castro-Alamancos (2004b)]. Recent work has shown that, in lightly anesthetized rats, cells in Pr5 have multiwhisker receptive fields just like cells in the VPM (Minnery and Simons, 2003), and thus Pr5 responses can fully account for the multiwhisker receptive fields found in the VPM (Minnery et al., 2003; Timofeeva et al., 2004).

Because Pr5 responses can account for the large receptive fields of VPM neurons, do multiwhisker responses increase in the VPM during activation because they are increasing in Pr5 or can these changes occur at the level of the thalamus without any change in Pr5? The present study found that a selective thalamic activation caused by application of carbachol in the VPM enhanced multiwhisker responses. Moreover, recordings from Pr5 cells revealed that activation caused by RF stimulation caused no change in Pr5 receptive fields while enlarging VPM receptive fields. Thus, this leads to the conclusion that the thalamus is suppressing AW responses during quiescent states and enhances those responses during activation. It is also noteworthy that during other conditions (e.g., very deep anesthesia), the trigeminal complex may also affect the size of VPM receptive fields. Pr5 is in a privileged location to do so. Indeed, some studies have found small receptive fields in Pr5 (Chiaia et al., 2000), but it seems that under our conditions, it is not necessary to trigger any trigeminal mechanism to enlarge the receptive fields of VPM cells during activation. During quiescent states, the receptive fields of Pr5 neurons are already large, as shown for lightly anesthetized animals (Minnery and Simons, 2003). This observation is not unexpected because at the level of temporal processing, Pr5 neurons are also able to follow high-frequency sensory inputs during quiescent states (urethane anesthesia), whereas VPM neurons cannot (Ahissar et al., 2000). Similarly, the low-pass filtering of sensory inputs in the VPM during quiescent states has been shown to be affected by selective thalamic activation (Castro-Alamancos, 2002b).

What may be the thalamic mechanisms that enhance AW responses during activation? There are two obvious candidates to consider: feedback inhibition from the reticular nucleus (nRt) and the level of depolarization of VPM neurons. These two factors have been shown to be instrumental in affecting the temporal response properties of VPM cells during activated states (CastroAlamancos, 2002b). The long-latency IPSPs from nRt are well suited to modulate the long-latency responses characteristic of AWs. These IPSPs begin as early as $5 \mathrm{~ms}$ after a whisker deflection and can last up to $200 \mathrm{~ms}$ during quiescent states (CastroAlamancos, 2002b). One possibility is that recurrent inhibition during quiescent states would lead to single-whisker receptive fields in the VPM. Indeed, in lightly anesthetized animals, when VPM cells respond to multiple whiskers, recurrent inhibition from the nRt is present in the VPM but seems to be a weak phenomenon, as determined using single-unit extracellular recordings (Minnery et al., 2003). This may be attributable to the fact that during activated states, VPM IPSPs driven by whisker deflections are reduced (Castro-Alamancos, 2002a,b). Thus, 
strong recurrent inhibition in the VPM may be responsible for the suppression of AW responses during quiescent states.

The other factor that may play an important role at the level of the thalamus to suppress AW responses during quiescent states is the level of depolarization of VPM neurons. If this plays a role, it would mean that AW EPSPs are smaller than PW EPSPs. Additional intracellular work is needed to explore this possibility. Thus, one scenario is that the excitatory responses of AWs emerge during activated states because of the postsynaptic depolarization and the reduction in recurrent inhibition of VPM cells during those states.

\section{Nonlinear summation of multiwhisker responses}

AW responses in the VPM are sensitive to previous stimulation of the PW and vice versa. Stimulation of an AW (or PW) suppresses the response to the PW (or AW) if the stimuli are delivered $30 \mathrm{~ms}$ apart (Simons and Carvell, 1989). The suppression tends to be stronger if the PW is the first (or conditioning) stimulus (Minnery et al., 2003). This fits well with the nonlinear summation (suppression) of simultaneous multiwhisker responses found in the present study. We found that the response resulting from stimulating the PW and five AWs simultaneously is very similar to the PW response alone, as if the AWs had not been stimulated. It appears as if the shorter-latency PW response suppresses all other longer-latency responses. Moreover, the nonlinear summation occurs both during quiescent states and during activation, suggesting that it does not require any of the changes that result from activation. However, the phenomenon is most obvious during activation because that is when the AW responses are stronger.

What may be suppressing AW responses when all whiskers are stimulated simultaneously? There seem to be two potential candidates. One possibility is that the suppression is caused by the recurrent inhibition from the nRt triggered by the PW response, which has a shorter latency. Alternatively, the suppression of AW responses may be caused at the level of Pr5. In this case, the thalamus would not have an active role in this nonlinear suppression and would simply be reflecting something that is occurring at the level of Pr5. Additional work will need to tease apart these possibilities.

\section{Functional implications}

Putting the results together, the study shows that thalamic activation enhances individual AWs responses (i.e., the excitatory surround of the receptive field) but suppresses those responses when both the PW and AWs are stimulated together. Thus, despite the increase in receptive field size, VPM cells represent only the center of the receptive field (PW) during simultaneous stimulation of multiple whiskers. Another interesting implication of the present study is that although VPM cells enlarge their receptive fields during activated states, it appears that at the next level of processing there is a focusing of receptive field size. That is, cells in the barrel cortex actually reduce their receptive fields during activation caused by receptive field stimulation (CastroAlamancos, 2002c). Indeed, in lightly anesthetized animals, barrel cortex neurons tend to respond mostly to the PW (Simons and Carvell, 1989; Goldreich et al., 1999). This spatial contrast at the level of the cortex appears to be caused by local recurrent inhibition (Simons, 1985, 1995; Miller et al., 2001; Pinto et al., 2003). An intriguing possibility is that the larger receptive fields in the VPM during activated states may actually favor selectivity in the barrel cortex by recruiting stronger recurrent inhibition.

\section{References}

Ahissar E, Sosnik R, Haidarliu S (2000) Transformation from temporal to rate coding in a somatosensory thalamocortical pathway. Nature 406:302-306.

Armstrong-James M, Callahan CA (1991) Thalamo-cortical processing of vibrissal information in the rat. II. spatiotemporal convergence in the thalamic ventroposterior medial nucleus (VPm) and its relevance to generation of receptive fields of S1 cortical "barrel” neurones. J Comp Neurol 303:211-224.

Berg RW, Friedman B, Schroeder LF, Kleinfeld D (2005) Activation of nucleus basalis facilitates cortical control of a brain stem motor program 2 . J Neurophysiol 94:699-711.

Bernardo KL, Woolsey TA (1987) Axonal trajectories between mouse somatosensory thalamus and cortex. J Comp Neurol 258:542-564.

Brecht M, Sakmann B (2002) Whisker maps of neuronal subclasses of the rat ventral posterior medial thalamus, identified by whole-cell voltage recording and morphological reconstruction. J Physiol (Lond) 538:495-515.

Brecht M, Preilowski B, Merzenich MM (1997) Functional architecture of the mystacial vibrissae. Behav Brain Res 84:81-97.

Carvell GE, Simons DJ (1990) Biometric analyses of vibrissal tactile discrimination in the rat. J Neurosci 10:2638-2648.

Castro-Alamancos MA (2002a) Properties of primary sensory (lemniscal) synapses in the ventrobasal thalamus and the relay of high-frequency sensory inputs. J Neurophysiol 87:946-953.

Castro-Alamancos MA (2002b) Different temporal processing of sensory inputs in the rat thalamus during quiescent and information processing states in vivo. J Physiol (Lond) 539:567-578.

Castro-Alamancos MA (2002c) Role of thalamocortical sensory suppression during arousal: focusing sensory inputs in neocortex. J Neurosci 22:9651-9655.

Castro-Alamancos MA (2004a) Absence of rapid sensory adaptation in neocortex during information processing states. Neuron 41:455-464.

Castro-Alamancos MA (2004b) Dynamics of sensory thalamocortical synaptic networks during information processing states. Prog Neurobiol 74:213-247.

Castro-Alamancos MA, Oldford E (2002) Cortical sensory suppression during arousal is due to the activity- dependent depression of thalamocortical synapses. J Physiol (Lond) 541:319-331.

Chiaia NL, Rhoades RW, Bennett-Clarke CA, Fish SE, Killackey HP (1991) Thalamic processing of vibrissal information in the rat. I. Afferent input to the medial ventral posterior and posterior nuclei. J Comp Neurol 314:201-216.

Chiaia NL, Zhang S, Crissman RS, Rhoades RW (2000) Effects of neonatal axoplasmic transport attenuation on the response properties of vibrissaesensitive neurons in the trigeminal principal sensory nucleus of the rat 1 . Somatosens Mot Res 17:273-283.

Deschenes M, Timofeeva E, Lavallee P (2003) The relay of high-frequency sensory signals in the whisker-to-barreloid pathway. J Neurosci 23:6778-6787.

Diamond ME, Armstrong-James M, Budway MJ, Ebner FF (1992) Somatic sensory responses in the rostral sector of the posterior group (POm) and in the ventral posterior medial nucleus (VPM) of the rat thalamus: dependence on the barrel field cortex. J Comp Neurol 319:66-84.

Friedberg MH, Lee SM, Ebner FF (1999) Modulation of receptive field properties of thalamic somatosensory neurons by the depth of anesthesia. J Neurophysiol 81:2243-2252.

Goldreich D, Kyriazi HT, Simons DJ (1999) Functional independence of layer IV barrels in rodent somatosensory cortex. J Neurophysiol 82:1311-1316.

Guic-Robles E, Valdivieso C, Guajardo G (1989) Rats can learn a roughness discrimination using only their vibrissal system. Behav Brain Res 31:285-289.

Henderson TA, Jacquin M (1995) What makes subcortical barrels? In: Cerebral cortex, Vol 11 (Jones EG, Diamond IT, eds), pp 123-187. New York: Plenum.

Jacquin MF, Woerner D, Szczepanik AM, Riecker V, Mooney RD, Rhoades RW (1986) Structure-function relationships in rat brainstem subnucleus interpolaris. I. Vibrissa primary afferents. J Comp Neurol 243:266-279.

Land PW, Buffer Jr SA, Yaskosky JD (1995) Barreloids in adult rat thalamus: 
three-dimensional architecture and relationship to somatosensory cortical barrels. J Comp Neurol 355:573-588.

Miller KD, Pinto DJ, Simons DJ (2001) Processing in layer 4 of the neocortical circuit: new insights from visual and somatosensory cortex. Curr Opin Neurobiol 11:488-497.

Minnery BS, Simons DJ (2003) Response properties of whisker-associated trigeminothalamic neurons in rat nucleus principalis. J Neurophysiol 89:40-56.

Minnery BS, Bruno RM, Simons DJ (2003) Response transformation and receptive field synthesis in the lemniscal trigeminothalamic circuit. J Neurophysiol 90:1556-1570.

Moore CI (2004) Frequency-dependent processing in the vibrissa sensory system 1. J Neurophysiol 91:2390-2399.

Moruzzi G, Magoun HW (1949) Brain stem reticular formation and activation of the EEG. Electroencephalogr Clin Neurophysiol 1:455-473.

Nicolelis MA, Chapin JK (1994) Spatiotemporal structure of somatosensory responses of many-neuron ensembles in the rat ventral posterior medial nucleus of the thalamus. J Neurosci 14:3511-3532.

Oldford E, Castro-Alamancos MA (2003) Input-specific effects of acetylcholine on sensory and intracortical evoked responses in the "barrel cortex" in vivo. Neuroscience 117:769-778.

Paxinos G, Watson C (1982) The rat brain in stereotaxic coordinates. New York: Academic.

Pinault D (1996) A novel single-cell staining procedure performed in vivo under electrophysiological control: morpho-functional features of juxtacellularly labeled thalamic cells and other central neurons with biocytin or Neurobiotin. J Neurosci Methods 65:113-136.

Pinto DJ, Hartings JA, Brumberg JC, Simons DJ (2003) Cortical damping: analysis of thalamocortical response transformations in rodent barrel cortex. Cereb Cortex 13:33-44.

Rhoades RW, Belford GR, Killackey HP (1987) Receptive-field properties of rat ventral posterior medial neurons before and after selective kainic acid lesions of the trigeminal brain stem complex. J Neurophysiol 57:1577-1600.
Shosaku A (1985) A comparison of receptive field properties of vibrissa neurons between the rat thalamic reticular and ventro-basal nuclei. Brain Res 347:36-40.

Simons DJ (1985) Temporal and spatial integration in the rat SI vibrissa cortex. J Neurophysiol 54:615-635.

Simons DJ (1995) Neuronal integration in the somatosensory whisker/barrel cortex. In: Cerebral cortex, Vol 11, The barrel cortex of rodents (Jones EG, Diamond IT, eds), pp 263-298. New York: Plenum.

Simons DJ, Carvell GE (1989) Thalamocortical response transformation in the rat vibrissa/barrel system. J Neurophysiol 61:311-330.

Steriade M, Jones EG, McCormick DA (1997) Thalamus. New York: Elsevier.

Timofeeva E, Lavallee P, Arsenault D, Deschenes M (2004) Synthesis of multiwhisker-receptive fields in subcortical stations of the vibrissa system. J Neurophysiol 91:1510-1515.

Vanderwolf CH (1988) Cerebral activity and behavior: control by central cholinergic and serotonergic systems. Int Rev Neurobiol 30:225-340.

Veinante P, Jacquin MF, Deschenes M (2000) Thalamic projections from the whisker-sensitive regions of the spinal trigeminal complex in the rat. J Comp Neurol 420:233-243.

Waite PM (1973) Somatotopic organization of vibrissal responses in the ventro-basal complex of the rat thalamus. J Physiol (Lond) 228:527-540.

Williams MN, Zahm DS, Jacquin MF (1994) Differential foci and synaptic organization of the principal and spinal trigeminal projections to the thalamus in the rat. Eur J Neurosci 6:429-453.

Woolsey TA, Van der Loos H (1970) The structural organization of layer IV in the somatosensory region (SI) of mouse cerebral cortex. The description of a cortical field composed of discrete cytoarchitectonic units. Brain Res 17:205-242.

Woolston DC, La Londe JR, Gibson JM (1982) Comparison of response properties of cerebellar- and thalamic-projecting interpolaris neurons 1 . J Neurophysiol 48:160-173. 